\title{
Temperature effect on acoustic plasmons
}

\author{
V. M. Silkin, ${ }^{1,2,3}$ V. U. Nazarov, ${ }^{4}$ A. Balassis, ${ }^{5}$ I. P. Chernov, ${ }^{6}$ and E. V. Chulkov ${ }^{1,2,7}$ \\ ${ }^{1}$ Departamento de Física de Materiales, Facultad de Ciencias Químicas, Universidad del País Vasco, \\ Apdo. 1072, 20080 San Sebastián/Donostia, Spain \\ ${ }^{2}$ Donostia International Physics Center (DIPC), Paseo de Manuel Lardizabal 4, 20018 San Sebastián/Donostia, Spain \\ ${ }^{3}$ IKERBASQUE, Basque Foundation for Science, 48011 Bilbao, Spain \\ ${ }^{4}$ Research Center for Applied Sciences, Academia Sinica, Taipei 11529, Taiwan \\ ${ }^{5}$ Department of Physics and Engineering Physics, Fordham University, 441 East Fordham Road, Bronx, New York 10458, USA \\ ${ }^{6}$ Tomsk Polytechnical University, pr. Lenina 30, 634050 Tomsk, Russia \\ ${ }^{7}$ Centro de Física de Materiales CFM-Materials Physics Center MPC, Centro Mixto CSIC-UPV/EHU, \\ Paseo de Manuel Lardizabal 5, 20018 San Sebastián/Donostia, Spain
}

(Received 18 March 2016; published 11 October 2016)

\begin{abstract}
The presence of several kinds of carriers at the Fermi surface results in interesting complex dielectric properties of the bulk Pd in the low-energy excitation range. A most spectacular manifestation of this is the presence of a collective electronic excitation characterized by a soundlike dispersion, termed acoustic plasmon (AP). Due to the characteristic dispersion reaching zero energy in the long-wavelength limit, the question of the thermal stability of the excitation spectrum arises. In this work we explore this problem investigating the thermal effect on the electronic excitation spectrum in this material, tracing how the AP properties vary with the temperature increase. The main effect consists in the gradual destruction of AP in the energy range corresponding to the temperature.
\end{abstract}

DOI: 10.1103/PhysRevB.94.165122

\section{INTRODUCTION}

In metallic systems the Fermi surface crossing by several energy bands with different Fermi velocities may result in a strong modification of dielectric properties as compared with those obtained for simple metals within a model of the homogeneous electron gas. One of the consequences may be the absence of an energy threshold for the collective electronic excitations [1-3], in contrast to a conventional free-electron three-dimensional gas model [4-6]. In particular, a collective excitation in which the slow carriers are dynamically screened by the fast ones can be realized [2]. Such a mode has a soundlike dispersion similar to that of the acoustic phonons, i.e., its energy decreases linearly to zero as the momentum goes to zero. Because of this peculiar dispersion this mode is frequently called acoustic plasmon (AP). During a long time the possibility of the existence of AP in real metals and its implications in physical phenomena such as superconductivity were continuously discussed [7-13], however, firm experimental or theoretical (taking into account full band structure effects) evidence of its existence was not presented. The key problem is that in metals the numerous intra- and interband single-particle electron-hole excitations may be very efficient in the destruction of such kind of collective electronic excitation in comparison with dilute electron systems in semiconducting heterostructures [14]. Consequently, a simplified theory like those employed for the description of collective electronic excitation in the near-free electron-gas systems cannot provide a definite proof of existence of AP in metals. Only recently, the detailed numerical calculations taking fully into account the realistic band structure predicted such kind of collective excitation in a variety of metal systems possessing several kinds of bulk carriers at the Fermi surface. In particular, it was predicted in elemental metals $\mathrm{Pd}[15,16]$ and $\mathrm{Pb}[17]$ and some layered materials like $\mathrm{MgB}_{2}$ [18], intercalated graphite
$\mathrm{CaC}_{6}$ [19], and transition-metal dichalcogenide $\mathrm{NbSe}_{2}$ [20]. Nevertheless, still such mode in bulk materials was not observed in energy-loss experiments, although a similar mode resulting in a two-component electron system at metal surfaces was detected [21-26].

The case of the AP in $\mathrm{Pd}$ is a particularly remarkable example. Calculations performed at zero temperature show that in this material, due to the presence [27-29] at the Fermi level of both the $s$ - $p$ - and the $d$-like energy bands (see Fig. 1) with distinct Fermi velocities, the AP should be a true three-dimensional feature characterized by a soundlike dispersion along all three main symmetry directions, spanning the energy interval from zero to about $1 \mathrm{eV}$ [15]. The absence of a low-energy threshold for its excitation poses an interesting question: how the AP properties may be affected by raising electronic temperature. It would be interesting to theoretically investigate this issue, in particular, in conjunction with the pump-probe optical experiments, since upon a pump pulse the electronic system may be quickly driven to a rather high temperature maintaining the ion lattice at low $T$ [30], and it is not clear whether AP mode would survive to be detected by the probe pulse.

In this paper we perform a detailed investigation of how the AP properties are varied upon the temperature change. In particular, we trace how the temperature modifies the real and imaginary parts of the dielectric function and observe the corresponding variations in the AP loss peak shape. In general, our calculations show that the increase of temperature results in the gradual degradation of the AP properties and its full destruction at high temperatures. At the same time, surprisingly, we find that the AP mode is a rather robust phenomenon against temperature increase and may exist even at rather elevated electron temperatures.

The rest of the paper is organized as follows. In Sec. II we describe details of the $a b$ initio calculation of the 


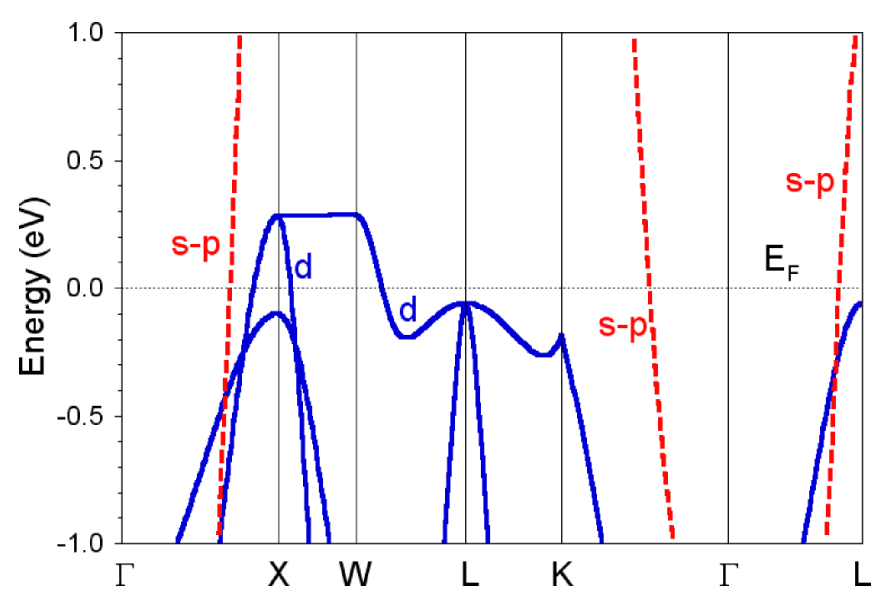

FIG. 1. Band structure of Pd in the vicinity of the Fermi level, $E_{F}$, set to zero. The energy bands of the $s-p$ and the $d$ character are shown by dashed and solid lines, respectively.

frequency- and momentum-dependent dielectric function in bulk solids at finite temperature. Results and their discussion are presented in Sec. III. The main conclusions of the present work are given in Sec. IV. Unless otherwise stated explicitly, atomic units ( $\left.\hbar=e^{2}=m_{e}=1\right)$ are used throughout the paper.

\section{CALCUlation METHOD}

We evaluate the excitation spectrum in Pd considering a fcc lattice with the lattice parameter $a_{c}=7.3512$ a.u. The central ingredients of its derivation - the one-particle energies $\varepsilon_{n \mathbf{k}}$ and wave functions $\psi_{n \mathbf{k}}(\mathbf{r})$ - were obtained from the selfconsistent solution of the Kohn-Sham equations using the band structure code [31]. The LDA exchange-correlation potential of Ref. [32] was employed. For the description of the electronion interaction we employed a nonlocal norm-conserving ionic pseudopotential [33].

A probability of the momentum $\mathbf{q}$ and energy $\omega$ transfer to the system is determined [5] by the imaginary part of the inverse dielectric function $\operatorname{Im}\left[\epsilon^{-1}(\mathbf{q}, \omega)\right]$, which in a periodic system takes a matrix form. Its evaluation was performed in the framework of the time-dependent density-functional theory $[34,35]$, where the dynamical density-response function $\chi\left(\mathbf{r}, \mathbf{r}^{\prime}, \omega\right)$ for interacting electrons obeys the integral equation $\chi=\chi^{0}+\chi^{0}\left(v+K^{\mathrm{xc}}\right) \chi, \chi^{0}\left(\mathbf{r}, \mathbf{r}^{\prime}, \omega\right)$ being the densityresponse function for noninteracting electrons and $K^{\mathrm{xc}}\left(\mathbf{r}, \mathbf{r}^{\prime}, \omega\right)$ accounting for dynamical exchange-correlation effects. Since in Pd, like in other transition metals [36], the low-energy excitation spectrum at small q's is insensitive to the shape of $K_{\mathrm{xc}}[15,16]$, in this work for its description we employed the random-phase approximation, i.e., setting $K_{\mathrm{xc}}=0$. Having evaluated the matrix $\chi_{\mathbf{G G}^{\prime}}(\mathbf{q}, \omega)$ constructed over the plane waves with 51 reciprocal vectors $\mathbf{G}$, the inverse dielectric function matrix $\epsilon_{\mathbf{G G}^{\prime}}^{-1}(\mathbf{q}, \omega)$ was obtained as

$$
\epsilon_{\mathbf{G G}^{\prime}}^{-1}(\mathbf{q}, \omega)=\delta_{\mathbf{G G}^{\prime}}+v_{\mathbf{G}}(\mathbf{q}) \chi_{\mathbf{G G}^{\prime}}(\mathbf{q}, \omega),
$$

where $v_{\mathbf{G}}(\mathbf{q})=4 \pi /|\mathbf{q}+\mathbf{G}|^{2}$ is the Fourier transform of the Coulomb potential.
The expression for the imaginary part of the $\chi^{0}$ function has the form

$$
\begin{aligned}
\operatorname{Im}\left[\chi_{\mathbf{G G}^{\prime}}^{0}(\mathbf{q}, \omega)\right]= & \frac{2}{\Omega} \sum_{\mathbf{k}}^{\mathrm{BZ}} \sum_{n n^{\prime}}\left(f_{n \mathbf{k}}-f_{n^{\prime} \mathbf{k}+\mathbf{q}}\right)\left\langle\psi_{n \mathbf{k}}\right| e^{-i(\mathbf{q}+\mathbf{G}) \cdot \mathbf{r}} \\
& \times\left|\psi_{n^{\prime} \mathbf{k}+\mathbf{q}}\right\rangle\left\langle\psi_{n^{\prime} \mathbf{k}+\mathbf{q}}\left|e^{i\left(\mathbf{q}+\mathbf{G}^{\prime}\right) \cdot \mathbf{r}}\right| \psi_{n \mathbf{k}}\right\rangle \\
& \times \delta\left(\varepsilon_{n \mathbf{k}}-\varepsilon_{n^{\prime} \mathbf{k}+\mathbf{q}}+\omega\right),
\end{aligned}
$$

where the factor 2 accounts for spin, sum over wave vectors $\mathbf{k}$ is performed in the first Brillouin zone (BZ), $n$ and $n^{\prime}$ are the energy-band indices, and $f_{n \mathbf{k}}$ are the temperature-dependent Fermi occupation factors. The real part of $\chi^{0}$ was obtained from $\operatorname{Im}\left[\chi^{0}\right]$ via the Kramers-Kronig relation by numerical integration. For this purpose we calculated the $\operatorname{Im}\left[\chi^{0}\right]$ matrices on a discrete mesh of energies from 0 to $10 \mathrm{eV}$ with a step of 2 meV using our homemade code [37]. The $\delta$ function in Eq. (2) was represented by a Gaussian with a broadening parameter of $5 \mathrm{meV}$. Although up to $51 \mathbf{G}$ vectors were included in the expansion of $\chi^{0}, \chi, \epsilon$, and $\epsilon^{-1}$ (in such a way including the local-field effects $[38,39])$, the final results for the calculated loss functions in the energy-momentum phase space of interest here are almost identical to the case when the local-field effects are neglected. In order to properly describe the fine details in the evaluated quantities, we employed a dense $240 \times 240 \times$ $240 \mathbf{k}$ mesh for summation in Eq. (2) over the BZ. Such mesh allowed us to trace the AP dispersion down to an energy of $\approx 30 \mathrm{meV}$.

\section{CALCULATION RESULTS AND DISCUSSION}

For a collective plasmon mode to occur in an electron system with the given transfer of the momentum $\mathbf{q}$ and the energy $\omega_{p}$, the energy-loss function

$$
-\operatorname{Im} \frac{1}{\epsilon(\mathbf{q}, \omega)}=-\operatorname{Im} \epsilon_{\mathbf{G}=0 \mathbf{G}^{\prime}=0}^{-1}(\mathbf{q}, \omega)
$$

must exhibit a maximum at $\omega=\omega_{p}$, which means that the dielectric function $\epsilon\left(\mathbf{q}, \omega_{p}\right)$ should (exactly or approximately) satisfy the equation

$$
\epsilon\left(\mathbf{q}, \omega=\omega_{p}\right)=0 .
$$

Whereas in a free-electron-gas model Eq. (4) has a solution for real energies $\omega$ in a certain momentum region [5], in real materials this almost never can be fulfilled exactly, but rather can hold approximately by both the real and imaginary parts of the dielectric function approaching zero. In such way, for a collective mode to exist at a certain energy $\omega_{p}$, the following three conditions should be fulfilled in the energy interval in the vicinity of $\omega_{p}$ : (a) a clear peak in the loss function must be present, (b) $\operatorname{Re}[\epsilon]$ should become or approach zero, and (c) a local minimum in $\operatorname{Im}[\epsilon]$ must be reached. In the subsequent analysis we will follow this scheme.

In Fig. 2 we present the imaginary part of the calculated normalized inverse dielectric function, $L(\mathbf{q}, \omega) \equiv$ $-\operatorname{Im}\left[\epsilon_{\mathbf{G}=0 \mathbf{G}^{\prime}=0}^{-1}(\mathbf{q}, \omega)\right] / \omega q$, for the momentum transfers along three main symmetry directions. These data are obtained for zero temperature. In the figure the dominant peaks which have a soundlike linear dispersion at small $q$ 's and represent the AP modes are highlighted by solid lines. In the $\langle 100\rangle$ and 

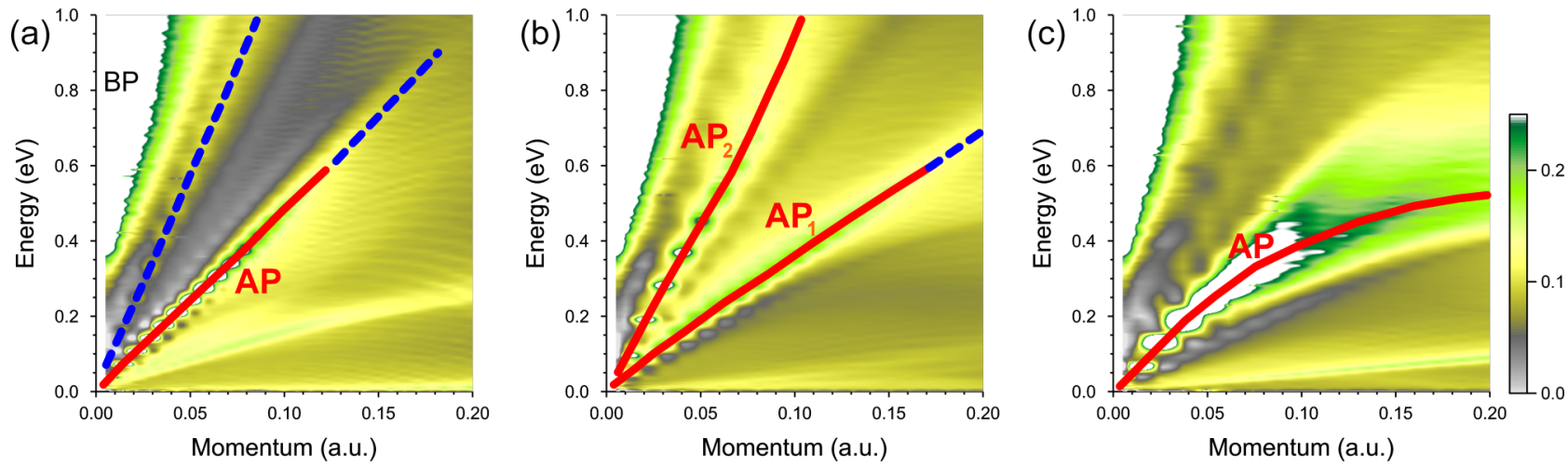

FIG. 2. Calculated normalized loss function, $L(\mathbf{q}, \omega)$, in Pd at $T=0$ versus the energy $\omega$ and the momentum transfer $\mathbf{q}$ along three main symmetry directions: (a) $\langle 100\rangle$, (b) $\langle 110\rangle$, and (c) $\langle 111\rangle$. Red solid lines highlight the dispersion of the AP modes. Blue dashed lines mark features corresponding to an enhanced number of single-particle excitations. A prominent feature in the upper-left corner in each panel [denoted as "BP" in (a)] corresponds to a low-energy tail of the bulk plasmon peak [40-43].

$\langle 111\rangle$ directions one can observe a single AP peak. Its origin is explained by the presence, as seen in Fig. 1, of the $s$ - $p$ - and $d$-like energy bands crossing the Fermi level with very different Fermi velocities [15]. As a result, an incomplete dynamical screening of the slow carriers in the $d$-like energy bands by the faster ones in the $s p$-like band leads to the appearance of such kind of collective excitation $[1,3,11,12]$. On the contrary, as seen in Fig. 2(b), along the $\langle 110\rangle$ direction there are two such modes dispersing with different group velocities. The presence of two AP modes instead of the expected one was explained by the existence of three groups of carriers (instead of two groups) at the Fermi level, moving with different Fermi velocities in this direction [15].

In this work the excitation spectrum of Pd was calculated for several values of the electronic temperature $T$ entering Eq. (2) through the temperature-dependent Fermi distribution function $f_{n \mathbf{k}}=1 /\left(e^{\left(\varepsilon_{n \mathbf{k}}-\mu\right) / T}+1\right)$, where $\mu$ is the chemical potential determined self-consistently by keeping the number of particles for each temperature at the band-structure-calculation level. In Fig. 3 we compare the dielectric function $\epsilon$ and the loss function $\operatorname{Im}\left[\epsilon^{-1}\right]$ calculated for $T=0,50,100,200$, and $500 \mathrm{meV}$ at $\mathbf{q}=0.021$ a.u. along the $\langle 100\rangle$ symmetry direction. In accord with the previous calculations $[15,16]$, here one can observe that at $T=0$ in this energy range $\operatorname{Im}[\epsilon]$ consists of two dominant peaks $I_{1}$ and $I_{2}$ centered at the energies of 27 and $160 \mathrm{meV}$, which are separated by a wide deep valley at energies between 90 and $140 \mathrm{meV}$, the latter marked by an arrow. The origin of such a shape of $\operatorname{Im}[\epsilon]$, as mentioned above, is linked to the presence at the Fermi level of two kinds of carriers moving in the $\langle 100\rangle$ crystal direction with very different group velocities [15]. As a result of such two-peak structure of the imaginary part of $\epsilon$, its real part presents a characteristic behavior with crossing the zero axis three times, in contrast to the conventional one-component free-electron-gas (FEG) picture [5] where such zero crossing occurs only once in this energy region. The first and third zero crossings of $\operatorname{Re}[\epsilon]$ almost coincide in energy with the peak positions in $\operatorname{Im}[\epsilon]$ resulting in an overdamping of the corresponding collective modes as occurs in the FEG model. On the contrary, since [as shown by an arrow in Fig. 3(b)] the second zero crossing of $\operatorname{Re}[\epsilon]$ occurs at $105 \mathrm{meV}$, i.e., at an energy where $\operatorname{Im}[\epsilon]$ has a shallow local minimum [highlighted by an arrow in Fig. 3(a)], a well-defined sharp peak denoted as AP appears in the corresponding loss function in Fig. 3(c) at nearly the same energy. The presence of such a peak in the loss function signals that the corresponding mode is a well-defined collective excitation. In the particular case of $T=0$, the linewidth of the loss peak is about $3 \mathrm{meV}$ corresponding to the mode lifetime of about $220 \mathrm{fs}$. In combination with the group velocity of this mode of about 0.184 a.u. [15], the propagation length of such an excitation may be as large as 1700 a.u., i.e., more than ten times larger than its wavelength of about

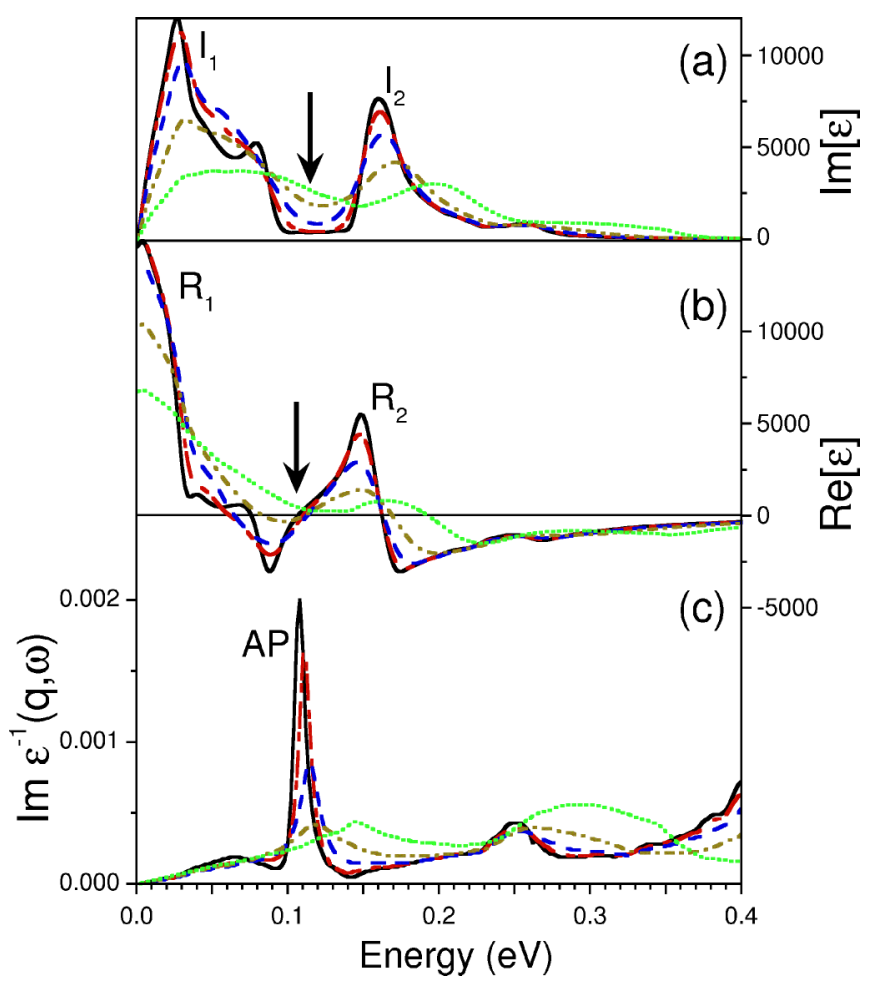

FIG. 3. Calculated (a) imaginary and (b) real parts of $\epsilon$ and (c) loss function, $\operatorname{Im}\left[\epsilon^{-1}\right]$, versus the energy $\omega$ at $q=0.021$ a.u. along the $\langle 100\rangle$ symmetry direction. Solid, dash-long-dashed, dashed, dashdotted, and dotted lines stand for the data calculated for $T=0,50$, 100,200 , and $500 \mathrm{meV}$, respectively. 
150 a.u. at this q. These estimations confirm that such collective electronic excitation in Pd is a well-defined quantity.

In Fig. 3 one can observe how the increase of the electronic temperature results in the modification of $\operatorname{Im}[\epsilon]$, consisting in lowering the peak amplitudes and smearing out of the valleys between them. Obviously, the main effect is observed on the lower-energy side, although even at $T=50 \mathrm{meV}$ a visible reduction of the height of the upper $I_{2}$ peak in $\operatorname{Im}[\epsilon]$ can be noticed. The reduction of the peak's height is accompanied by the transmission of spectral weight to the higher-energy side, in order to fulfill the $f$-sum-rule condition. Such evolution of $\operatorname{Im}[\epsilon]$ produces the corresponding gradual smoothing of the peak structure in $\operatorname{Re}[\epsilon]$ as well. In particular, the height of the peak $R_{2}$ at $150 \mathrm{meV}$ reduces very quickly upon the $T$ increase, whereas this effect becomes clearly visible in the height of the lower-energy peak $R_{1}$ at temperatures above $50 \mathrm{meV}$.

One can notice in Fig. 3 that the reduction of the height of the two main peaks $I_{1}$ and $I_{2}$ in $\operatorname{Im}[\epsilon]$ with the temperature increase is accompanied by a blue shift of its energy positions. On the contrary, the position of the first peak $R_{1}$ in $\operatorname{Re}[\epsilon]$ is anchored at $\omega=0$ at all the temperatures. The same insensitivity to the temperature is observed for the energy position of the peak $R_{2}$ in $\operatorname{Re}[\epsilon]$ in Fig. 3(b) up to $T=$ $200 \mathrm{meV}$. Only at $T=500 \mathrm{meV}$ one can see how this peak shows a shift toward higher energies by about $25 \mathrm{meV}$ in comparison with the $T=0$ case. An opposite trend is observed for a negative part of $\operatorname{Re}[\epsilon]$ which has a local minimum at low temperatures around $\omega \approx 90 \mathrm{meV}$, which lifts up upon a temperature increase. Such trend results in that at a certain

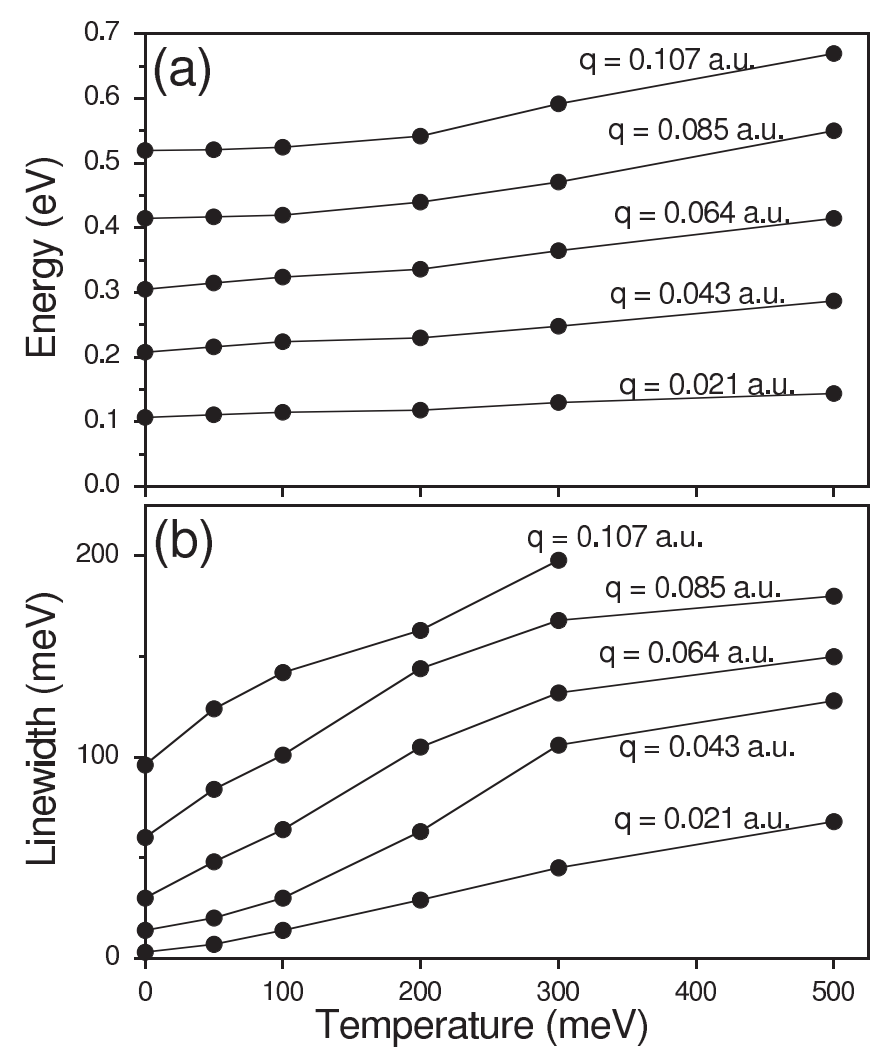

FIG. 4. Calculated (a) energy and (b) linewidth of the AP peak at some values of momentum $q$ directed along the $\langle 100\rangle$ axis. temperature the two lowest-energy zero crossings of $\operatorname{Re}[\epsilon]$ cease to occur. In this particular case these no zero crossings are observed in the case of curves calculated at $T=500 \mathrm{meV}$. As seen in Fig. 3(c) this leads to a dramatic reduction of the strength of the AP peak in the loss function signaling that this mode becomes overdamped, decaying very efficiently into incoherent electron-hole pairs. However, even at such elevated temperature, the peak in the loss function in Fig. 3(c) is still visible at $\omega=145 \mathrm{meV}$. On the other hand, the heating up to $200 \mathrm{meV}$ does not change the collective nature of this mode at this $\mathbf{q}$, although the width of the loss peak at $T=$ $200 \mathrm{meV}$ is increased by a factor of 10 . From this observation, we conclude that the AP can be a well-defined collective excitation with energies notably lower than the temperature at which the electronic system is maintained.

It is corroborated by Figs. 4(a) and 4(b) where the energy and linewidth, respectively, of the AP peak at some momentum $q$ values along the $\langle 100\rangle$ symmetry direction are reported. For instance, at $T=0$ the linewidth ranges from $3 \mathrm{meV}$ at $q=0.021$ a.u. to $96 \mathrm{meV}$ at $q=0.107$ a.u., whereas the energy of this mode varies from 107 to $520 \mathrm{meV}$. Since the linewidth is significantly smaller than its energy, this mode can be considered as a well-defined collective excitation. On the other hand, in Fig. 4 it is seen how upon the temperature increase the AP mode energy experiences the blue shift accompanied by a gradual linewidth increase. As a result, at $T=500 \mathrm{meV}$ the linewidth becomes comparable with

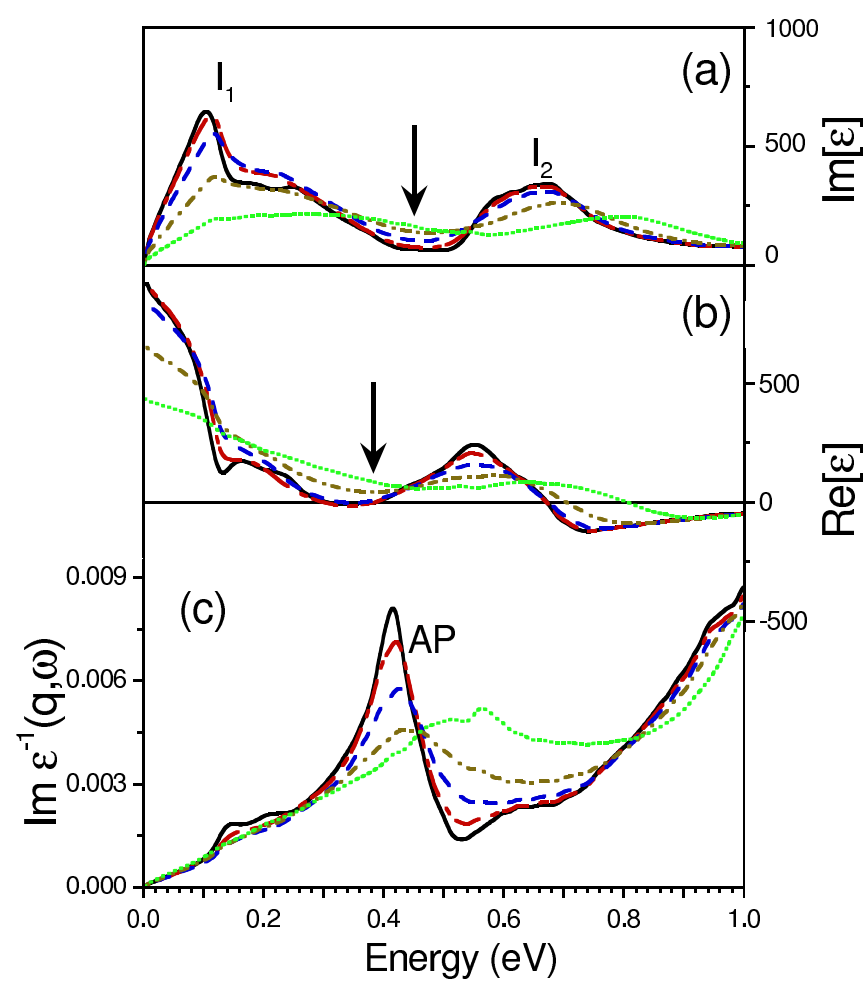

FIG. 5. Calculated (a) imaginary and (b) real parts of $\epsilon$ and (c) loss function, $\operatorname{Im}\left[\epsilon^{-1}\right]$, versus energy $\omega$ at $q=0.085$ a.u. along the $\langle 100\rangle$ symmetry direction. Solid, dash-long-dashed, dashed, dash-dotted, and dotted lines stand for the data calculated for $T=0,50,100,200$, and $500 \mathrm{meV}$, respectively. 

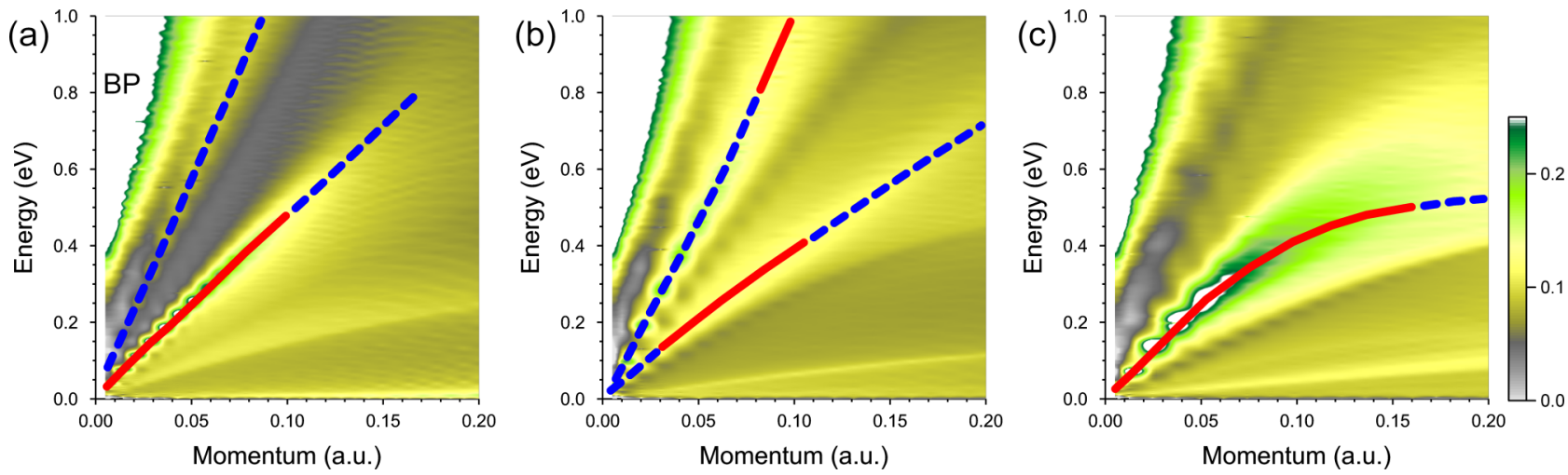

FIG. 6. Calculated normalized loss function, $L(\mathbf{q}, \omega)$, in Pd at $T=100 \mathrm{meV}$ versus the energy $\omega$ and momentum transfer $\mathbf{q}$ along three main symmetry directions: (a) $\langle 100\rangle$, (b) $\langle 110\rangle$, and (c) $\langle 111\rangle$. Red solid lines highlight the dispersion of the AP modes. Blue dash lines mark features corresponding to the enhanced number of single-particle excitations.

the energy and, consequently, the AP mode ceases to be a well-defined collective excitation.

Observing Fig. 3, one can notice that a mere fact of presence of two separate peaks in $\operatorname{Im}[\epsilon]$ does not guarantee the existence of a collective mode in the in-between energy interval. For instance, in Fig. 3(a) one can see two peaks in $\operatorname{Im}[\epsilon]$ even at $T=500 \mathrm{meV}$. However, the shape and positions of these peaks are not favorable to force $\operatorname{Re}[\epsilon]$ to cross the zero axis in the energy interval between these two peaks. Consequently, at this temperature only a very broad peak at $\omega=145 \mathrm{meV}$ in the loss function can be detected, which cannot be interpreted as a collective mode. It consists mainly of the incoherent electronhole pair excitations.

A similar mechanism of disappearance of the AP mode is observed at $T=0$ upon the momentum increase. Thus, as seen in Fig. 5(b), at $q=0.085$ a.u. along the $\langle 100\rangle$ direction the real part of $\epsilon$ after dropping below zero at $\omega=320 \mathrm{meV}$ quickly jumps to the positive values, crossing zero the second time at $400 \mathrm{meV}$. As a result, even at $T=0$, the AP peak width in the loss function is significantly enhanced in comparison with the case of $q=0.021$ a.u. of Fig. 3(c). In Fig. 5(a) one can observe that the increase of temperature smears out a two-peak $I_{1}-I_{2}$ structure in $\operatorname{Im}[\epsilon]$. This is accompanied by an upward shift in energy of the plasmon AP peak in the loss function of Fig. 5(c) together with its increasing broadening due to decay into the electron-hole pairs. If the magnitude of $q$ increases more, the AP becomes quickly overdamped and loses completely its collective nature at $q>0.12$ a.u. as shown in Fig. 2(a) by a dashed line.

Additionally, in Fig. 3(a) at zero temperature one can notice a weak peak at $\omega=80 \mathrm{meV}$. However, its strength is not sufficient to produce a well-defined separate peak in the loss function at lower energies. Instead, a broad weak feature is seen in the loss function of Fig. 3(c) around $\omega=65 \mathrm{meV}$. This single-particle peak can be observed as a dispersing feature in the lower part of Fig. 2(a). Upon the temperature increase, this peak quickly loses its intensity in accord with the disappearance of a separate peak in $\operatorname{Im}[\epsilon]$, seen in Fig. 3(c). A similar effect can be observed in the upper-energy side of the AP peak. At temperatures below $200 \mathrm{meV}$, one can discern a peak in the loss function at the energy of $250 \mathrm{meV}$. At higher temperatures it shifts to larger energies. Its origin can be traced to the presence of the shallow weak peak in $\operatorname{Im}[\epsilon]$ at $\omega \approx 265 \mathrm{meV}$, which also shifts to higher energies upon the temperature increase. However, since the real part of the dielectric function does not cross zero in the vicinity of this peak, the corresponding mode does not have a collective nature. Indeed, this weak peak in $\operatorname{Im}\left[\epsilon^{-1}\right]$ persists at all small $q$ 's and its dispersion is shown by the upper dashed line in Fig. 2(a).
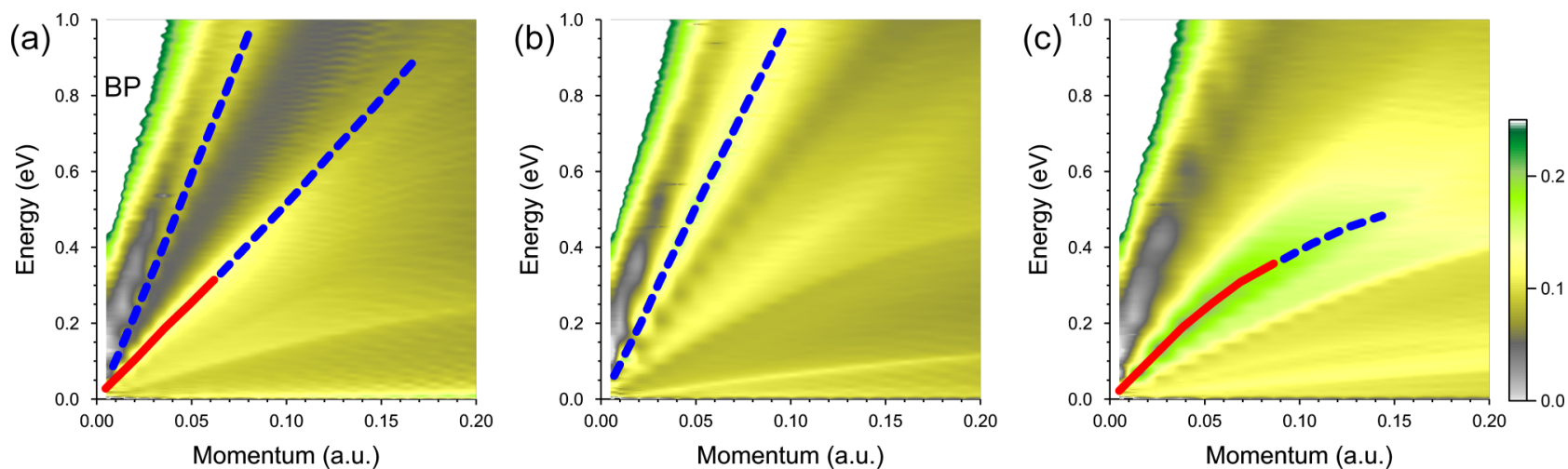

FIG. 7. Calculated normalized loss-function, $L(\mathbf{q}, \omega)$, in $\mathrm{Pd}$ at $T=200 \mathrm{meV}$ versus the energy $\omega$ and momentum transfer $\mathbf{q}$ along three main symmetry directions: (a) $\langle 100\rangle$, (b) $\langle 110\rangle$, and (c) $\langle 111\rangle$. Red solid lines highlight the dispersion of the AP modes. Blue dashed lines mark features corresponding to an enhanced number of single-particle excitations. 

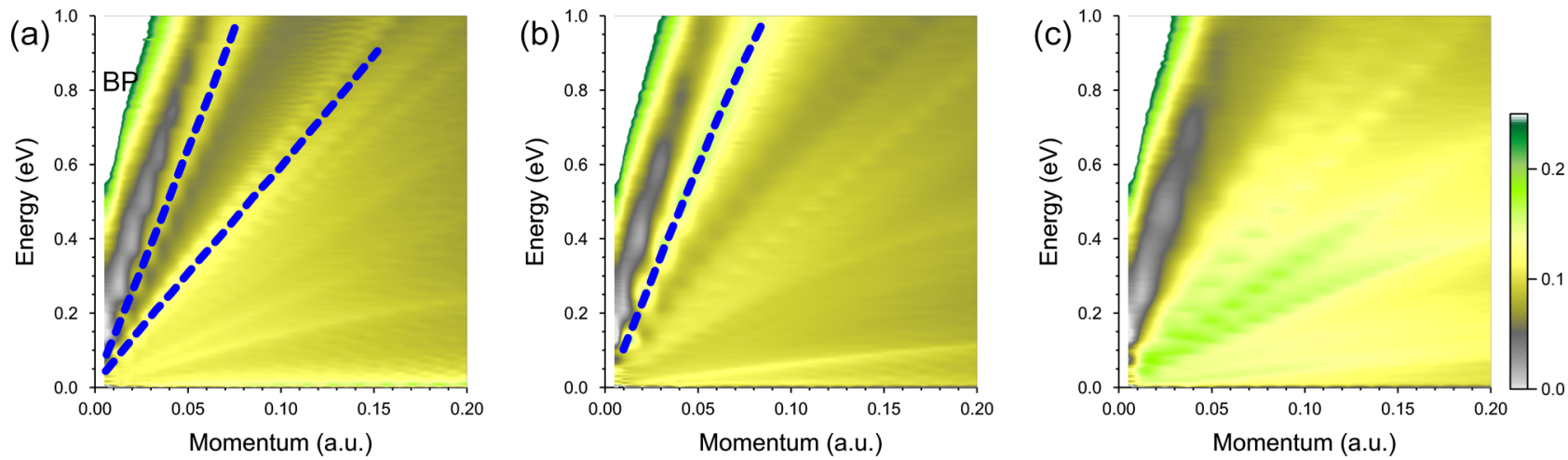

FIG. 8. Calculated normalized loss function, $L(\mathbf{q}, \omega)$, in $\mathrm{Pd}$ at $T=500 \mathrm{meV}$ versus the energy $\omega$ and momentum transfer $\mathbf{q}$ along three main symmetry directions: (a) $\langle 100\rangle$, (b) $\langle 110\rangle$, and (c) $\langle 111\rangle$. Blue dashed lines mark features corresponding to the enhanced number of single-particle excitations.

As was found in Refs. [15,16], along the $\langle 110\rangle$ direction two AP modes are presented. The corresponding peaks in the lossfunction are highlighted in Fig. 2(b) by solid lines. A careful analysis of the character of the lower $\mathrm{AP}_{1}$ mode confirms that it has a collective nature up to the energy of about $0.6 \mathrm{eV}$, whereas the upper $\mathrm{AP}_{2}$ mode is well defined up to energies well above $1 \mathrm{eV}$ [16].

Comparing Fig. 2(a) with Fig. 2(c), one can observe that the AP mode has initial group velocity in the $\langle 111\rangle$ direction very similar to that in the $\langle 100\rangle$ direction. However, at the momentum transfer exceeding 0.05 a.u. in the $\langle 111\rangle$ direction, the dispersion of this mode starts to deviate from the linear law significantly. At the same time it maintains its collective behavior within a more extended momentum transfer interval, being a well-defined collective feature up to $q=0.2$ a.u. in contrast to the case of Fig. 2(a), where the AP mode loses its collective nature at $q$ 's beyond $q \approx 0.12$ a.u.

Figures 6-8 present the normalized loss function of Pd evaluated at $T=100, T=200$, and $T=500 \mathrm{meV}$, respectively. Again, as in Fig. 2, well-defined AP peaks are highlighted by solid lines and prominent single-particle peaks by dashed lines. From comparison of the data for the $\langle 100\rangle$ and $\langle 111\rangle$ directions, one can deduce that the increase of temperature results in the destruction of the AP peak starting from the upper-energy part, in contrast to our expectation. For instance, at $T$ as high as $200 \mathrm{meV}$, the AP mode can be distinguished in Figs. 7(a) and 7 (c) down to $\sim 30$ and $\sim 50 \mathrm{meV}$, respectively. Clearly, the temperature increase results in the AP peak broadening as demonstrated in Figs. 3 and 9 for small $q$ 's along the $\langle 100\rangle$ and $\langle 111\rangle$ directions, respectively. How the energy and the linewidth of the AP peak for $q$ directed along the $\langle 111\rangle$ axis evolve with temperature variation can be seen in Fig. 10. The existence of the AP mode at energies significantly lower than the temperature can be explained by the fact that the carrier velocities in Pd are joined in the well-separated groups well far away from the Fermi surface, as can be seen in Fig. 3 of Ref. [15]. Consequently, even at elevated temperatures, this grouping helps to maintain the separate-peaks structure in $\operatorname{Im}[\epsilon]$, like it was found in graphene [44].

The temperature dependence of the low-energy plasmon dispersion along the $\langle 110\rangle$ direction is more complicated. From the comparison of $L(\mathbf{q}, \omega)$ at $T=100 \mathrm{meV}$ in Fig. 6(b) with the zero-temperature case of Fig. 2(b) one can see that the lowerenergy $\mathrm{AP}_{1}$ mode starts to dilute from both the upper- and the lower-energy sides. In particular, at $T=100 \mathrm{meV}$ this mode loses its collective character at energies below $\omega \sim 100 \mathrm{meV}$. In more detail, this can be seen for $q=0.02$ a.u. in Fig. 11 . Even though three clear peaks $I_{1}, I_{2}$, and $I_{3}$ can be resolved at $T=100 \mathrm{meV}$ in $\operatorname{Im}[\epsilon]$ of Fig. 11(a), its real part crosses zero only once at $\omega \approx 55 \mathrm{meV}$. At other zero crossings observed at lower temperatures the real part at this $T$ drops below the zero line. Moreover, $\operatorname{Re}[\epsilon]$ is almost flat in the energy interval where the $\mathrm{AP}_{1}$ mode exists at low temperature. As a result, only very broad structure is seen at $T=100 \mathrm{meV}$ in the loss function in Fig. 11(c) at $\omega$ in the $50-150 \mathrm{meV}$ interval. In the case of the upper-energy $\mathrm{AP}_{2}$ mode the effect of $T$ is not so

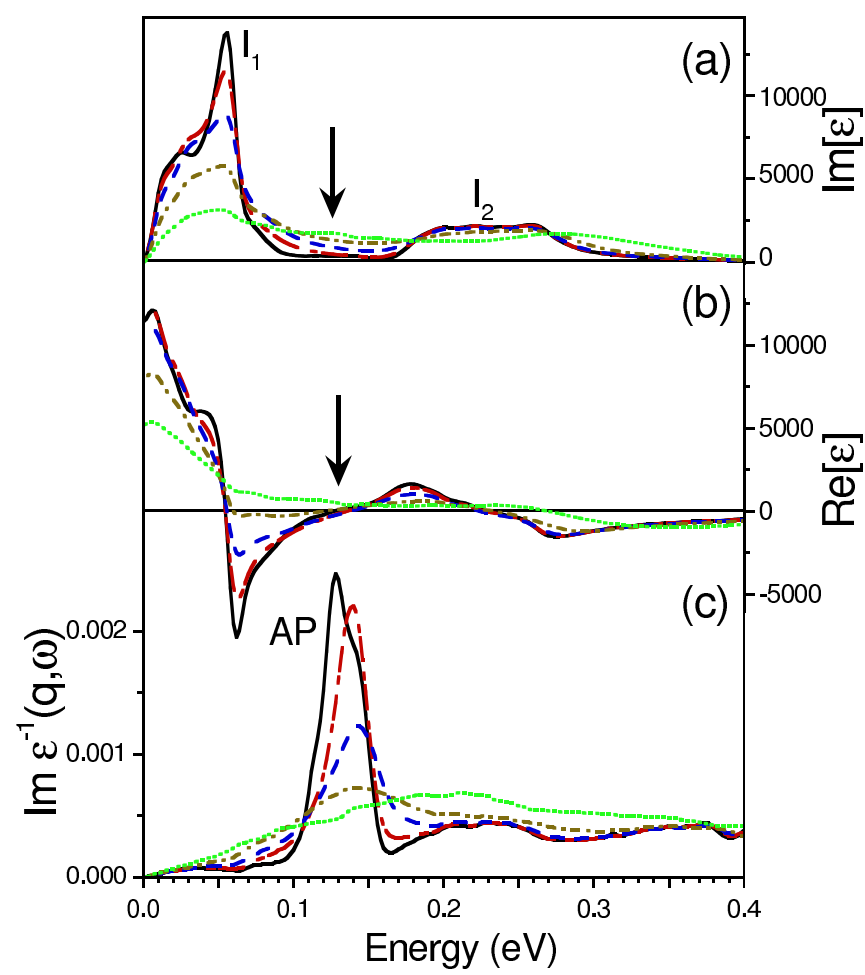

FIG. 9. Calculated (a) imaginary and (b) real parts of $\epsilon$ and (c) loss function, $\operatorname{Im}\left[\epsilon^{-1}\right]$, versus the energy $\omega$ at $q=0.025$ a.u. along the 〈111 symmetry direction. Solid, dash-long-dashed, dashed, dashdotted, and dotted lines stand for the data calculated for $T=0,50$, 100,200 , and $500 \mathrm{meV}$, respectively. 


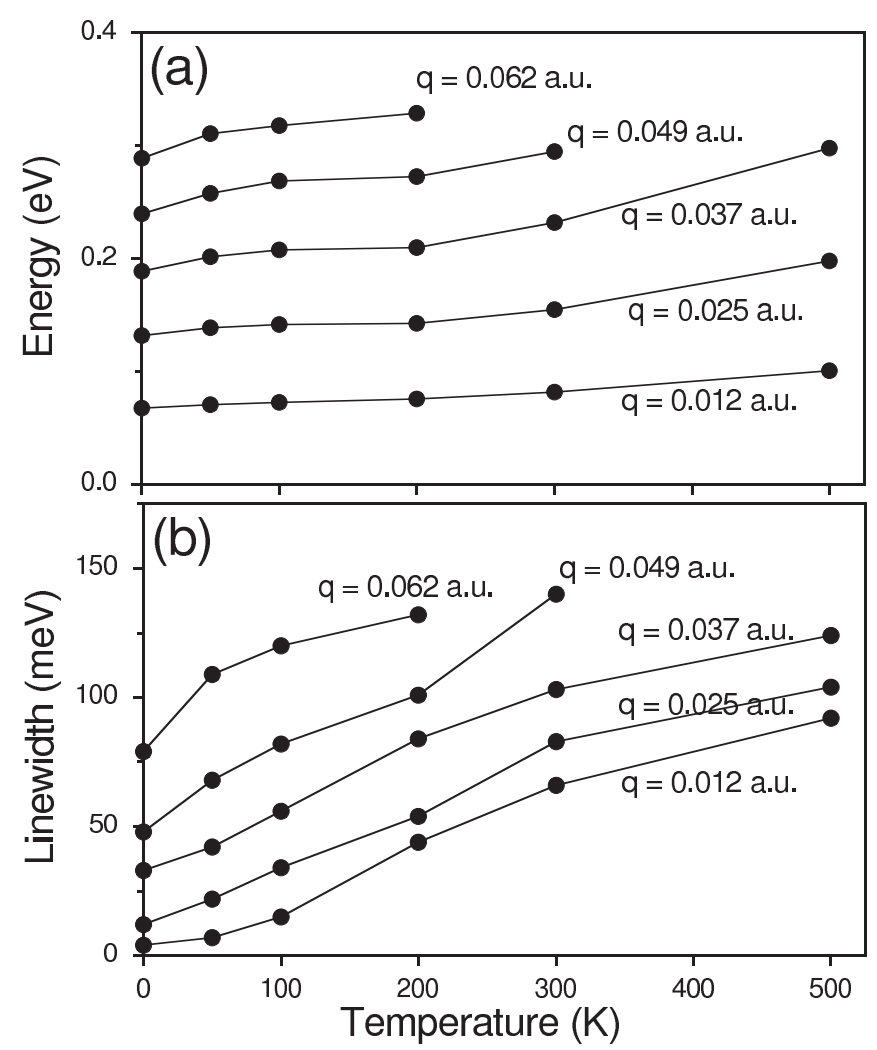

FIG. 10. Calculated (a) energy and (b) linewidth of the AP peak at some values of momentum $q$ directed along the $\langle 111\rangle$ axis.

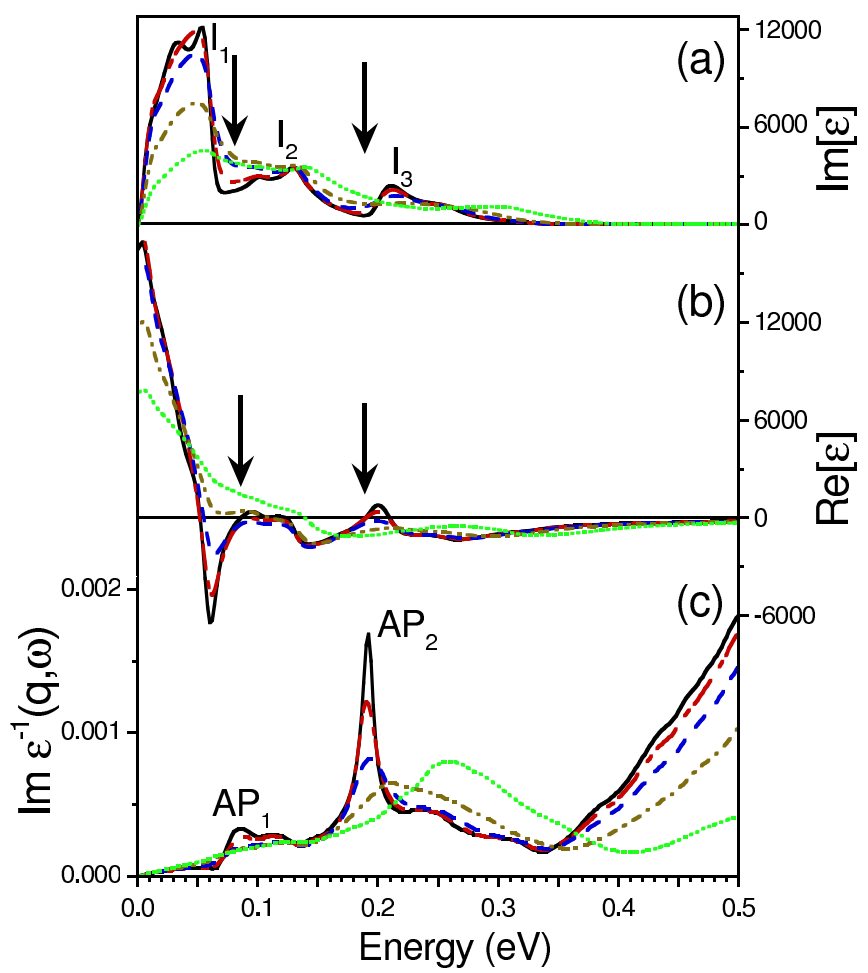

FIG. 11. Calculated (a) imaginary and (b) real parts of $\epsilon$ and (c) loss function, $\operatorname{Im}\left[\epsilon^{-1}\right]$, versus the energy $\omega$ at $q=0.020$ a.u. along the $\langle 110\rangle$ symmetry direction. Solid, dash-long-dashed, dashed, dashdotted, and dotted lines stand for the data calculated for $T=0,50$, 100, 200, and $500 \mathrm{meV}$, respectively. dramatic, and a rather well-defined peak in the loss function can be found at $T=100 \mathrm{meV}$ at this $q$. However, since at this temperature $\operatorname{Re}[\epsilon]$ is maintained below the zero line in its vicinity, we interpret it as a single-particle excitation.

On the other hand, upon the temperature increase the upper AP mode starts to disappear from the lower-energy side. Thus, at $T=100 \mathrm{meV}$ the $\mathrm{AP}_{2}$ mode presents itself as a collective mode at the energies above $0.8 \mathrm{eV}$.

From Figs. 7(a) and 7(c) it is clear that at $T=200 \mathrm{meV}$ the regions in the momentum-energy phase space where the AP can exist reduces significantly. Nevertheless, the scenario of its disappearance from the upper-energy side still holds, and this mode still has clear collective character down to the lowest accessible energies. At the same time, along the $\langle 110\rangle$ direction the lower-energy $\mathrm{AP}_{1}$ ceases to exist at this $T$, whereas the upper-energy $\mathrm{AP}_{2}$ peak is transformed into a broad singleparticle peak denoted in Fig. 7(b) by a dashed line. Even though at such temperature, as follows from Fig. 12, the $\mathrm{AP}_{2}$ peak linewidth in the low-energy interval is several times smaller than its energy, the absence of a corresponding zero crossing in $\operatorname{Re}[\epsilon]$ does not allow us to interpret it as a collective excitation.

As seen in Fig. 8(c), the increasing temperature up to $T=$ $500 \mathrm{meV}$ results in a complete disappearance of the AP in the $\langle 111\rangle$ direction. At the same time, even at such elevated temperature, notable peaks in the loss function traced to the AP at low temperatures can be detected in two other symmetry directions. They are clearly shifted upward in comparison with the $T=0$ case of Fig. 2 .

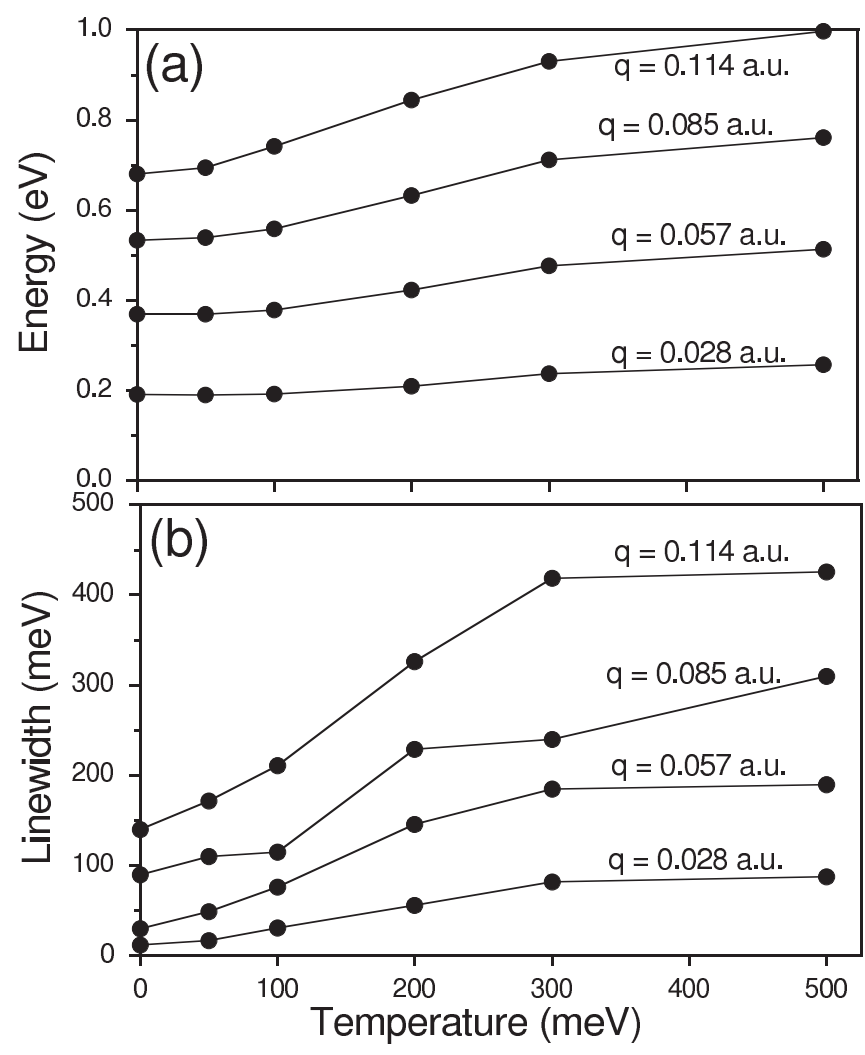

FIG. 12. Calculated (a) energy and (b) linewidth of the upperenergy $\mathrm{AP}_{2}$ peak at some values of momentum $q$ directed along the $\langle 110\rangle$ axis. 


\section{CONCLUSIONS}

In this work, we have investigated the evolution of the low-energy collective electronic excitations in bulk Pd with variation of the electronic temperature. It was found that the acoustic plasmon modes are rather robust excitations against the temperature increase. Thus they can be observed at energies significantly lower in comparison with the electron temperature. Moreover, in contrast to our expectations, the extinction of the AP modes upon the temperature increase is observed generally from the higher-energy side, whereas in the lower-energy side the temperature effect at moderate $T$ 's is limited to the AP linewidth increase. Such scenario is realized for the momentum transfers along the $\langle 100\rangle$ and $\langle 111\rangle$ symmetry directions. Along the $\langle 110\rangle$ direction, this is accompanied by the gradual extinction of the lower-energy AP mode as well.
Our findings confirm that the AP mode can be realized in this material at rather elevated electron temperature. At the same time the temperature increase may introduce a notable upward shift of the AP dispersion. We believe that these findings may constitute an interesting topic for an experimental verification performed, for instance, in the pump-probe optical experiments on the $\mathrm{Pd}$ thin films in infrared interval.

\section{ACKNOWLEDGMENTS}

V.M.S. and E.V.C. acknowledge partial support from the University of the Basque Country, Grant No. IT-756-13, and the Spanish Ministry of Economy and Competitiveness MINECO, Grant No. FIS2013-48286-C2-1-P. V.U.N. acknowledges support from the Ministry of Science and Technology, Taiwan, Grants No. 104-2112-M-001-007 and No. 105-2112-M-001-010.
[1] D. Pines, Can. J. Phys. 34, 1379 (1956).

[2] P. Nozières and D. Pines, Phys. Rev. 109, 1062 (1958).

[3] D. Pines and J. R. Schrieffer, Phys. Rev. 124, 1387 (1961).

[4] J. Lindhard, Mat.-Fys. Medd. - K. Dan. Vidensk. Selsk. 28, 1 (1954).

[5] D. Pines and P. Nozières, The Theory of Quantum Liquids: Normal Fermi Liquids (W. A. Benjamin, New York, 1966), Vol. 1.

[6] G. Giuliani and G. Vignale, Quantum Theory of the Electron Liquid (Cambridge University Press, Cambridge, 2009).

[7] B. T. Geilikman, Sov. Phys. JETP 21, 796 (1965).

[8] H. Fröhlich, J. Phys. C 1, 544 (1968).

[9] J. Ruvalds, Adv. Phys. 30, 677 (1981).

[10] Y. Ishii and J. Ruvalds, Phys. Rev. B 48, 3455 (1993).

[11] R. F. Wood, B. E. Sernelius, and A. L. Chernyshev, Phys. Rev. B 66, 014513 (2002).

[12] A. Bill, H. Morawitz, and V. Z. Kresin, Phys. Rev. B 66, 100501(R) (2002).

[13] V. Z. Kresin and S. A. Wolf, Rev. Mod. Phys. 81, 481 (2009).

[14] T. Ando, A. B. Fowler, and F. Stern, Rev. Mod. Phys. 54, 437 (1982).

[15] V. M. Silkin, I. P. Chernov, Yu. M. Koroteev, and E. V. Chulkov, Phys. Rev. B 80, 245114 (2009).

[16] V. M. Silkin, V. U. Nazarov, I. P. Chernov, I. Yu. Sklyadneva, and E. V. Chulkov, J. Phys.: Condens. Matter 27, 055501 (2015).

[17] X. Zubizarreta, V. M. Silkin, and E. V. Chulkov, Phys. Rev. B 87, 115112 (2013).

[18] V. M. Silkin, A. Balassis, P. M. Echenique, and E. V. Chulkov, Phys. Rev. B 80, 054521 (2009).

[19] J. P. Echeverry, E. V. Chulkov, P. M. Echenique, and V. M. Silkin, Phys. Rev. B 85, 205135 (2012).

[20] M. N. Faraggi, A. Arnau, and V. M. Silkin, Phys. Rev. B 86, 035115 (2012).

[21] V. M. Silkin, A. García-Lekue, J. M. Pitarke, E. V. Chulkov, E. Zaremba, and P. M. Echenique, Europhys. Lett. 66, 260 (2004).

[22] B. Diaconescu, K. Pohl, L. Vattuone, L. Savio, Ph. Hofmann, V. M. Silkin, J. M. Pitarke, E. V. Chulkov, P. M. Echenique, D. Farías, and M. Rocca, Nature (London) 448, 57 (2007).
[23] S. J. Park and R. E. Palmer, Phys. Rev. Lett. 105, 016801 (2010).

[24] K. Pohl, B. Diaconescu, G. Vercelli, L. Vattuone, V. M. Silkin, E. V. Chulkov, P. M. Echenique, and M. Rocca, Europhys. Lett. 90, 57006 (2010).

[25] R. Díez Muiño, D. Sánchez-Portal, V. M. Silkin, E. V. Chulkov, and P. M. Echenique, Proc. Natl. Acad. Sci. USA 108, 971 (2011).

[26] A. Politano and G. Chiarello, Prog. Surf. Sci. 90, 144 (2015).

[27] N. V. Smith, Phys. Rev. B 9, 1365 (1974).

[28] N. E. Christensen, Phys. Rev. B 14, 3446 (1976).

[29] C. T. Chan and S. G. Louie, Phys. Rev. B 27, 3325 (1983).

[30] W. S. Fann, R. Storz, H. W. K. Tom, and J. Bokor, Phys. Rev. B 46, 13592 (1992).

[31] V. M. Silkin, E. V. Chulkov, I. Yu. Sklyadneva, and V. E. Panin, Izv. Vyssh. Uchebn. Zaved., Fiz. 9, 56 (1984) [Sov. Phys. J. 27, 762 (1984)].

[32] D. M. Ceperley and B. J. Alder, Phys. Rev. Lett. 45, 566 (1980); as parametrized by J. P. Perdew and A. Zunger, Phys. Rev. B 23, 5048 (1981).

[33] N. Troullier and J. L. Martins, Phys. Rev. B 43, 1993 (1991).

[34] M. Petersilka, U. J. Gossmann, and E. K. U. Gross, Phys. Rev. Lett. 76, 1212 (1996).

[35] E. Runge and E. K. U. Gross, Phys. Rev. Lett. 52, 997 (1984).

[36] I. G. Gurtubay, J. M. Pitarke, W. Ku, A. G. Eguiluz, B. C. Larson, J. Tischler, P. Zschack, and K. D. Finkelstein, Phys. Rev. B 72, 125117 (2005).

[37] V. M. Silkin, E. V. Chulkov, and P. M. Echenique, Phys. Rev. B 68, 205106 (2003).

[38] S. L. Adler, Phys. Rev. 126, 413 (1962).

[39] N. Wiser, Phys. Rev. 129, 62 (1963).

[40] E. G. Maksimov, I. I. Mazin, S. N. Rashkeev, and Yu. A. Uspenski, J. Phys. F 18, 833 (1988).

[41] G.-M. Fehrenbach, Phys. Rev. B 59, 15085 (1999).

[42] E. E. Krasovskii and W. Schattke, Phys. Rev. B 63, 235112 (2001).

[43] V. M. Silkin, I. P. Chernov, P. M. Echenique, Yu. M. Koroteev, and E. V. Chulkov, Phys. Rev. B 76, 245105 (2007).

[44] E. H. Hwang and S. Das Sarma, Phys. Rev. B 79, 165404 (2009). 